\section{Two-photon excited autofluorescence imaging of human retinal pigment epithelial cells}

\author{
Meng Han* \\ University of Heidelberg \\ Faculty of Clinical Medicine \\ Mannheim Biomedical Engineering Laboratories \\ and \\ Kirchhoff Institute for Physics \\ Heidelberg, Germany
}

\author{
Almut Bindewald-Wittich \\ Frank G. Holz \\ University of Bonn \\ Department of Ophthalmology \\ Bonn, Germany
}

\section{Guenter Giese}

Max-Planck-Institute for Medical Research

Heidelberg, Germany

\author{
Markolf H. Niemz \\ University of Heidelberg \\ Faculty of Clinical Medicine \\ Mannheim Biomedical Engineering Laboratories \\ Heidelberg, Germany
}

\author{
Sarah Snyder \\ Hui Sun \\ Jiayi Yu \\ Michael Agopov \\ Olivier La Schiazza \\ Josef F. Bille \\ University of Heidelberg \\ Kirchhoff Institute for Physics \\ Heidelberg, Germany
}

\begin{abstract}
Degeneration of retinal pigment epithelial (RPE) cells severely impairs the visual function of retina photoreceptors. However, little is known about the events that trigger the death of RPE cells at the subcellular level. Twophoton excited autofluorescence (TPEF) imaging of RPE cells proves to be well suited to investigate both the morphological and the spectral characteristics of the human RPE cells. The dominant fluorophores of autofluorescence derive from lipofuscin (LF) granules that accumulate in the cytoplasm of the RPE cells with increasing age. Spectral TPEF imaging reveals the existence of abnormal LF granules with blue shifted autofluorescence in RPE cells of aging patients and brings new insights into the complicated composition of the LF granules. Based on a proposed twophoton laser scanning ophthalmoscope, TPEF imaging of
\end{abstract}

*Tel: +49-6221-549254; E-mail: mhan@urz.uni-hd.de the living retina may be valuable for diagnostic and pathological studies of age related eye diseases. () 2006 Society of Photo-Optical Instrumentation Engineers. [DOI: 10.1117/1.2171649]

Keywords: multiphoton; microscopy; retina; autofluorescence.

Paper 05330LRR received Nov. 4, 2005; revised manuscript received Dec. 9, 2005 and Dec. 27, 2005; accepted for publication Dec. 28, 2005; published online Feb. 10, 2006.

Aged-related macular degeneration (AMD) is the principal cause of irreversible loss of vision and registered legal blindness for aging people in developed countries. To place this in perspective, $35 \%$ of the human population over the age of 75 has some degree of AMD. ${ }^{1,2}$ AMD is characterized by the progressive degeneration of retina, RPE cells, and choroid. The earliest visible abnormality in AMD is the accumulation of drusen (waste material) between the RPE cells and the choroid. ${ }^{1}$ Despite considerable research, little is known about the events that trigger AMD. One of the hypotheses widely accepted among ophthalmologists suggests that the degeneration of RPE is responsible for drusen formation. ${ }^{1}$ However, how the RPE cells are damaged with increasing age is not clear. Previous studies proposed that LF granules, a byproduct of incomplete digestions of the distal ends of photoreceptors, ${ }^{3}$ are most likely harmful when present in sufficient amount in the RPE cells. ${ }^{4}$ Since LF granules in the RPE cells demonstrate strong autofluorescence (AF) when excited by blue/ green light, a number of pioneering works have focused on the characteristics of the AF from the living retina with either ophthalmic or spectrophotometric fundus imaging. ${ }^{5-10}$ Here, we are more interested in the morphological and spectral features of individual LF granules in RPE cells, which may provide fresh insights into the pathological cause of RPE cell death at the subcellular level.

The advent of femtosecond lasers led to the experimental implementation of two-photon laser scanning microscopy. ${ }^{11,12}$ Since two-photon absorption is confined within the central focus of the illuminating laser beam, diffraction-limited resolution and depth discrimination are achievable without extra pinholes, permitting 3D optical sectioning of thick tissues. Instead of ultraviolet (UV) or blue light typically employed for confocal fluorescence imaging, infrared (IR) laser can be used, providing deeper sensing depth with reduced phototoxic or beaching effects outside of the laser focus. ${ }^{13-16}$ Due to the transparency of the human eye to IR light, two-photon cornea or retina imaging has tremendous potential in developing novel diagnostic methods for ophthalmology. Recently, the fine structures of the collagen fibrils in cornea and sclera have been successfully resolved with second harmonic imaging microscopy. ${ }^{17-20}$ However, to the best of our knowledge, there are no reports of two-photon excited fluorescence (TPEF) imaging of individual human RPE cells with relevant discussions of the feasibility of a two-photon laser scanning ophthalmoscope.

In this study, TPEF imaging was performed on an upright (Zeiss LSM 510 NLO) laser scanning microscope equipped with a femtosecond Ti:sapphire laser (Coherent Chameleon $\mathrm{XR}$ ). To minimize potential photodamage to the RPE cells, the excitation laser power is attenuated to $4 \mathrm{~mW}$. A 63 $\times(\mathrm{N} . \mathrm{A} .=1.0)$ water immersion objective (Zeiss) was utilized for TPEF imaging. Through a set of dichroic beamsplit-

1083-3668/2006/11(1)/010501/3/\$22.00 @ 2006 SPIE 


\section{JBO LETTERS}

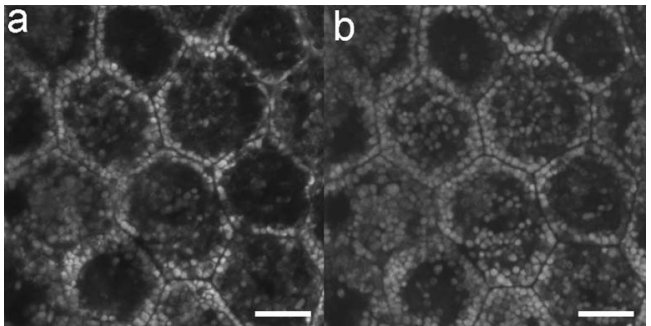

Fig. 1 Confocal (a) and TPEF (b) imaging of human RPE cells in the macula of a 55-year-old donor eye. AF of LF granules in RPE cells were excited either by (a) an argon laser $\left(\lambda_{A r}=488 \mathrm{~nm}\right)$ or (b) a femtosecond Ti:sapphire laser $\left(\lambda_{T i S}=800 \mathrm{~nm}\right)$, respectively. AF emission wavelength: (a) $\lambda_{A F}>560 \mathrm{~nm}$, (b) $500 \mathrm{~nm}<\lambda_{A F}<550 \mathrm{~nm}$. Bar: $10 \mu \mathrm{m}$.

ters, the AF signals were detected by two photomultiplier tubes (PMT) assigned to different spectral windows. The AF spectrum of LF granules in RPE cells was measured by a confocal laser scanning microscope (Leica TCS SP2) coupled to a UV argon ion laser $(\lambda=364 \mathrm{~nm})$. The AF in the wavelength range of 400-700 $\mathrm{nm}$ can be measured with a spectral resolution of $5 \mathrm{~nm}$ through a motor-driven slit in front of PMT detectors. The human retinas were obtained from three postmortem donor eyes $(19,55$, and 80 years old, with normal vision) from the eye hospital, University of Bonn, Germany. The posterior half of the eyeball was cut and fixed with paraformaldehyde (4\% in PBS, pH 7.4). A $5 \mathrm{~mm}$ diameter retina/sclera tissue probe was prepared with a surgical trephine. After the neurosensory retina was removed, the tissue probe was transferred to a custommade tissue dish filled with 4\% PBS ( $\mathrm{pH} 7.4)$ and was imaged with a water immersion objective.

As revealed in Fig. 1, the predominant fluorophores of AF in RPE cells were distributed in the LF granules. LF granules were preferably located adjacent to the cell membranes, which nicely outlined the shapes of the RPE cells. Although the typical diameter of LF granules is less than $1 \mu \mathrm{m}$, the boundary of LF granules can be resolved by both confocal and TPEF imaging. In order to precisely determine the distribution of LF granules in their host RPE cells, the nuclei of the RPE cells were stained with 4'-6-Diamidino-2-phenylindole (DAPI). As demonstrated in Fig. 2, the cytoplasm between the cell membrane and the nuclei tended to be completely occupied by LF granules. Impressively, double cell nuclei were observed in certain RPE cells, which may correspond to de-

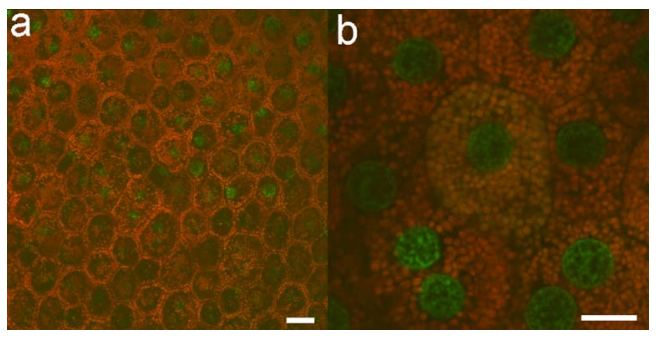

Fig. 2 TPEF imaging of RPE cells (55-year-old donor eye) with dual channel detection: red $\left(\lambda_{A F}>515 \mathrm{~nm}\right)$, green $\left(\lambda_{A F}=435-485 \mathrm{~nm}\right)$. Cell nuclei of RPE cells were stained with DAPI. Excitation wavelength: $\lambda_{T i S}=730 \mathrm{~nm}$. Bar: $10 \mu \mathrm{m}$.
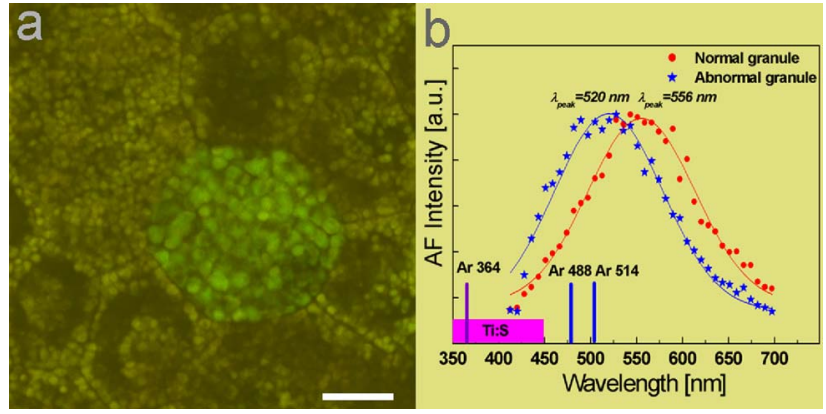

Fig. 3 (a) TPEF imaging of RPE cells (80-year-old donor eye) with dual channel detection: red $\left(\lambda_{A F}=575-640 \mathrm{~nm}\right)$, green $\left(\lambda_{A F}\right.$ $=500-550 \mathrm{~nm})$. Abnormal LF granules were recognized due to their blue shifted AF. Excitation wavelength: $\lambda_{T i S}=800 \mathrm{~nm}$. Bar: $10 \mu \mathrm{m}$. (b) The AF spectra of single LF granules which are representative were measured with 8-nm steps and were fitted with a Gaussian function. The blue stars and the red circles denote the AF spectra of abnormal and normal LF granules, respectively. Excitation wavelength: $\lambda_{A r}$ $=364 \mathrm{~nm}$. Wavelengths of typical excitation lasers are marked in the $x$ axis. (Ar: argon laser, Ti:S: Ti:sapphire laser).

generation of the RPE cell membranes. In addition to the established methods such as mass spectrometry and Western blot analysis, ${ }^{21,22}$ we can noninvasively probe the complicated composition of LF granules with spectral fluorescence imaging. The AF spectra of LF granules were first quantified with dual channel fluorescence detection. Different spectral windows were coded in colors. As shown in Fig. 3(a), the existence of abnormal LF granules exhibiting enhanced bluegreen fluorescence was immediately confirmed. Interestingly, most abnormal LF granules appear larger than normal LF granules and are confined within isolated single RPE cells. The abnormal LF granules exist only in the 55- and 80-yearold donor eyes and are not present in the 19-year-old eye. Since RPE cells containing abnormal LF granules are rare $(0.09 \%-0.12 \%)$, whether they are correlated to dysfunctions of RPE cells is beyond the scope of this report. Previous investigations revealed blue shifted AF from the Bruch's membrane in the AMD donor eyes, ${ }^{23}$ which was not observed in our study. In order to characterize the complete AF spectra of the LF granules, the same sample was measured with the Leica TCS SP2 confocal microscope, which is capable of performing simultaneous spectral profiling besides conventional $X Y$ scanning. As demonstrated in Fig. 3(b), AF spectra from single LF granules were measured with $8-\mathrm{nm}$ spectral resolution, which can be nicely fitted with a single Gaussian function. Since the typical emission lines of the argon laser $(\lambda$ $=488,514 \mathrm{~nm}$ ) are not sufficient to excite the complete AF spectra of RPE cells, an UV $(\lambda=364 \mathrm{~nm})$ argon laser has to be employed. The emission peak of the AF from abnormal LF granules is located at $520 \mathrm{~nm}$, which is significantly blue shifted compared with the fluorescence peak $(556 \mathrm{~nm})$ of normal LF granules.

With confocal and TPEF microscopic imaging, the morphological and spectral features of LF granules were quantitatively characterized. As age increases, LF granules accumulate in the RPE cells and in some cases nearly completely fill the cytoplasm. The typical diameters of LF granules are less than $1 \mu \mathrm{m}$, but a few RPE cells contain larger LF granules. Dual channel AF detection reveals a strong correlation be- 
tween the size and the AF spectra of these abnormal LF granules, whose AF peak is blue shifted from 556 to $520 \mathrm{~nm}$. As the fingerprint of the intracellular structures, abnormal AF implies the presence of new fluorophores or proteins in LF granules, which may indicate disturbed metabolic processes of the host RPE cells. Instead of utilizing multiline argon lasers for confocal imaging, TPEF imaging with a tunable Ti:sapphire laser turns out to be ideal to investigate both the morphological and spectral characteristics of RPE cells. Besides in vitro RPE cell imaging, TPEF seems valuable for in vivo retina imaging based on a two-photon laser scanning ophthalmoscope, which can be reconstructed from a conventional confocal retina tomographic scanner. First, since UV light cannot penetrate the cornea, only an IR beam is able to probe the complete AF spectra of the living retina. Second, as a second order process, the point spread function (PSF) of TPEF is approximately the square of the PSF of confocal imaging, thus the side lobes of the PSF due to the aberrations of the human eye are considerably suppressed. Spatial resolution can be improved without applying confocal pinholes or adaptive optics. However, since the cross section $(\sigma)$ of TPEF is usually much smaller than the corresponding single photon excitation process, the amplitude of the TPEF signals and the potential nonlinear photodamage in the laser focus have to be considered. The intensity of the fluorescence signals $F$ of single and two-photon excitation processes can be estimated by $F_{1} \propto \sigma_{1} I_{A r}$ and $F_{2} \propto \sigma_{2} I_{T i S}^{2}\left(\frac{T}{\tau}\right)$, where subscripts 1 and 2 denote the single photon and two-photon excited fluorescence, respectively, $I$ is the average fluence of the argon laser or the Ti:sapphire laser, $\tau$ is the pulse duration of the Ti:sapphire laser pulses (140 fs), and $T$ denotes the round-trip time of the femtosecond pulse in the laser cavity (11 ns). If assigning typical values of $\sigma_{2}$ of $10^{-50} / \mathrm{cm}^{4} s$ and $\sigma_{1}$ of $10^{-17} / \mathrm{cm}^{2}$ to the fluorophore in the LF granules, ${ }^{24} F_{2}$ is approximately 4 orders of magnitude less than $F_{1}\left(I_{A r}\right.$ and $\left.I_{T i S}=4 \mathrm{~mW}\right)$. However, since the retina can be exposed to more excitation power with IR illumination and a large active area detector with a high amplification rate can be employed, the weak TPEF signals from the living retina may still be detectable. Furthermore, considering the rapid progress in living cell labeling techniques, the cellular events in the retina may be unraveled by the fluorescent labels (organic dyes or fluorescent proteins) which have large two-photon absorption cross sections. Nevertheless, only through careful examinations with an appropriate animal model or an efficient fluorescent labeling method, can we eventually construct and test the performance of the two-photon laser scanning ophthalmoscope, which may improve not only the resolution, but also the sensitivity for diagnostic and pathogenetic study of age related retina diseases.

\section{References}

1. D. Bok, "New insights and new approaches toward the study of agerelated macular degeneration," Proc. Natl. Acad. Sci. U.S.A. 99, 14619-14621 (1999).

2. R. Klein, B. E. Klein, and K. L. Linton, Ophthalmology 99, 933-943 (1992).

3. R. W. Young and D. Bok, "Participation of the retinal pigment epithelium in the rod outer segment renewal process," J. Cell Biol. 42,
392-402 (1969).

4. F. G. Holz, F. Schuett, J. Kopitz, G. E. Eldred, J. E. Voelcker, and M. Cantz, "Inhibition of lysosomal degradative functions in RPE cells by a retinoid component of lipofuscin," Invest. Ophthalmol. Visual Sci. 40, 737-743 (1999).

5. F. C. Delori, C. K. Dorey, G. Staurenghi, O. Arend, D. G. Goger, and J. J. Weiter, "In vivo fluorescence of the ocular fundus exhibits retinal pigment epithelium lipofuscin characteristics," Invest. Ophthalmol. Visual Sci. 36, 718-729 (1995).

6. F. C. Delori, G. Douglas, D. G. Goger, and C. K. Dorey, "Age-related accumulation and spatial distribution of lipofuscin in RPE of normal subjects," Invest. Ophthalmol. Visual Sci. 42, 1855-1866 (2001).

7. S. Schmitz-Valckenberg, S. Bueltmann, J. Dreyhaupt, A. Bindewald, F. G. Holz, and K. Rohrschneider, "Fundus autofluorescence and fundus perimetry in the junctional zone of geographic atrophy in patients with age-related macular degeneration," Invest. Ophthalmol. Visual Sci. 45, 4470-4476 (2004).

8. A. Bindewald, J. J. Jorzik, A. Loesch, F. Schutt, and F. G. Holz, "Visualization of retinal pigment epithelial cells in vivo using digital high-resolution confocal scanning laser ophthalmoscopy," Am. J. Ophthalmol. 137, 556-558 (2004).

9. A. von Rückmann, F. W. Fitzke, and A. C. Bird, "Distribution of fundus autofluorescence with a scanning laser ophthalmoscope," $\mathrm{Br}$. J. Ophthamol. 79, 407-412 (1995).

10. C. Bellmann, G. S. Rubin, S. A. Kabanarou, A. C. Bird, and F. W. Fitzke, "Fundus autofluorescence imaging compared with different confocal scanning laser ophthalmoscopes," Br. J. Ophthamol. 87, 1381-1387 (2003).

11. W. Denk, J. H. Strickler, and W. W. Webb, "Two-photon laser scanning fluoresence microscopy," Science 248, 73-76 (1990).

12. B. R. Masters, Selected Papers on Multiphoton Excitation Microscopy, Milestone Series 175, SPIE Press, Bellingham, WA (2003).

13. R. M. Williams, D. W. Piston, and W. W. Webb, "Two-photon laser molecular excitation provides in trinsic 3-dimensional resolution for laser-based microscopy and microphotochemistry," FASEB J. 8, 804813 (1994).

14. W. R. Zipfel, R. M. Williams, and W. W. Webb, "Nonlinear magic: multiphoton microscopy in the biosciences," Nat. Biotechnol. 21, 1369-1377 (2003)

15. P. J. Campagnola, H. A. Clark, W. A. Mohler, A. Lewis, and L. M. Loew, "Second-harmonic imaging microscopy for visualizing biomolecular arrays in cells, tissues and organisms," Nat. Biotechnol. 21, 1356-1360 (2003).

16. K. Koenig, "Multiphoton microscopy in life sciences," J. Microsc. 200, 83-104 (2000).

17. A. T. Yeh, N. Nassif, A. Zoumi, and B. J. Tromberg, "Selective corneal imaging using combined second-harmonic generation and two-photon excited fluorescence," Opt. Lett. 27, 2082-2084 (2002).

18. M. Han, L. Zickler, G. Giese, F. Loesel, M. Walter, and J. Bille, "Second harmonic corneal imaging after femtosecond laser surgery," J. Biomed. Opt. 9, 760-766 (2004).

19. M. Han, G. Giese, L. Zickler, H. Sun, and J. F. Bille, "Mini-invasive corneal surgery and imaging with femtosecond lasers," Opt. Express 12, 4275-4281 (2004).

20. M. Han, G. Giese, and J. F. Bille, "Second harmonic generation imaging of collagen fibrils in cornea and sclera," Opt. Express 13, 5791-5795 (2005).

21. F. Schutt, B. Ueberle, B. Schnolzer, F. G. Holz, and J. Kopitz, "Proteome analysis of lipofuscin in human retinal pigment epithelial cells," FEBS Lett. 528, 217-221 (2002).

22. F. Schutt, M. Bergmann, F. G. Holz, and J. Kopitz, "Proteins modified by malondialdehyde, 4-hydroxynonenal, or advanced glycation end products in lipofuscin of human retinal pigment epithelium," Invest. Ophthalmol. Visual Sci. 44, 3663-3668 (2003).

23. A. D. Marmorstein, L. Y. Marmorstein, H. Sakaguchi, and J. G. Hollyfield, "Spectral profiling of autofluorescence associated with lipofuscin, Bruchs membrane, and sub-RPE deposits in normal and AMD eyes," Invest. Ophthalmol. Visual Sci. 43, 2435-2441 (2002).

24. C. Xu, R. M. Williams, W. Zipfel, and W. W. Webb, "Multiphoton excitation cross-sections of molecular fluorophores," Bioimaging $\mathbf{4}$, 198-207 (1996). 